\title{
SEMANTIC SHIFT TO PRAGMATIC MEANING IN SHARED DECISION MAKING: SITUATION THEORY PERSPECTIVE
}

\author{
M. CANAN, A. SOUSA-POZA \& S.F. KOVACIC \\ Engineering Management \& System Engineering, Old Dominion University, USA.
}

\section{ABSTRACT}

The way humans establish communication depends on the generation and conveyance of meaning. Linguistically, meaning in information is dependent on the meaning that is ascribed to signifiers in the context of the communication. These signifiers can include items such as words, phrases, signs, and symbols. Conveyance of meaning may, however, imprecise and prone to error.

The meaning of information in communication may arise from a change in the context in which a signifier is placed (intrinsic), or a change in the paradigm with which the signifier and context are perceived (extrinsic). In simple situations, where paradigms are reconcilable, semantic shift is solely intrinsic. In complex situations, where differing paradigms will generally lead to irreconcilable perspectives (paradoxes and dualities); the semantic shift will be both intrinsic and extrinsic. Decisions are based on an individual's (or individuals' shared) understanding and understanding is in turn contingent on perspective. Decision making will, therefore, be affected by discrepancies in meaning.

It is critical to understand the nature of the discrepancies where shared awareness is necessary to enable group decisions. The theoretical construct presented recognizes that (1) a semantic shift may be required where multiple perspectives based on different paradigms come into play and (2) a semantic shift may introduce error, inefficiency, noise or redundancy. Therefore, individual limits can be recognized via shared awareness, which can be studied with situation theory.

Keywords: semantic, pragmatics, situation theory, situational awareness, shared awareness, decision making.

\section{INTRODUCTION}

The universal definition of decision making, namely: "choosing among alternatives", renders decision making an essential component of daily life. The spectrum of daily life is comprehensive, constituted by a variety of systems, such as military, private business, governments, or families. The two salient common characteristics of these systems are that (i) the primary agents in these systems are human beings and (ii) all these systems constitute a communication system that allows an information flow among the communicative components, or agents. Communication depends on the generation and conveyance of meaning. Linguistically, meaning in information is dependent on the meaning that is ascribed to signifiers in the context of the communication. These signifiers can include items such as words, phrases, signs, and symbols.

The meaning of information in communication may arise from differences in the context in which a signifier is placed (intrinsic), or a change in the paradigm with which the signifier and context are perceived (extrinsic). Regardless of being intrinsic or extrinsic in terms of cause, the differences entail discrepancy in meaning, which entails an epistemological gap. Therefore, the best possible decision is contingent on this epistemic gap, which separates the objective individual claims from objective communal claims.

This work is not a linguistic study. Rather, the primary concern is how an efficient informative communication can be attained. A Situations Theory perspective is utilized where situation is defined as a set of conditions that human beings expand on with the requirement that an individual is or becomes cognizant of the set of conditions [1]. Situation in this context bifurcates in two states: simple and 
complex, where the demarcation between the two is constrained by comprehensibility and understanding of the described situation.

The social facts in human life are being discussed and studied with various disciplines with an inquiry of how human beings create social structures that are cooperative and productive. In this regard, information flow is important for human beings who commune in social structures with assertoric commitments that may need further justification for decision making. Therefore, social interaction fosters individual's need to form organizations that constitute the following salient dynamics: communication, understanding awareness, and decision making.

\section{COMMUNICATION}

Communication is not a mere act of imparting information via language. It constitutes components of assertoric commitments that are verified with social interaction. Real things and the assertoric individual claims about real things are opaque. Thus, further probing is necessary for individual and for communal understanding to develop. Human beings utilized language as a communication system. This system constitutes messages to be communicated, communicative agents, and a set of signifiers. An abstract example of communication in an organizational setting is that there is a sender who would like to convey an understanding to a receiver so that a shared awareness can be established between a sender and a receiver.

\subsection{Communication models}

Communication can be undertaken in many different ways. These include textbooks, public speeches, social media, telephone conversation, media, email, radio, TV, and so on. The models presented here constitutes three levels of communication: intrapersonal communication [2], public communication [3], and interpersonal communication.

The first two communication types are not representing the communal aspects of communication. However, these two form the basis for the interpersonal communication.

Communication models are a representation of certain aspects of events, structures, or system made by using symbols [4]. The salient communication models such as Lasswell [5], Shannon and Weaver [6], Berlo [7], Dance [8], Barnlund [8] conform a sender and a receiver interaction with different approaches. They all agree that, communication is dynamic, continuous, circular, unrepeatable, irreversible, and complex.

\subsection{Systemic model of communication}

Systemic approach to communication is utilized in modeling communication as a system. The characterization of communication with the General System Theory's primary principle is comprehension of the related parts so that a change in one aspect entails changes in all other parts [9]. The systemic approach to communication constitutes the following axioms introduced by Watzlawick and Jackson [10]; (i) the impossibility of not communicating; (ii) content and relationship in communication; (iii) the punctuation of the sequence of events; and (iv) symmetrical and complementary interaction.

\subsection{Language as a communication system}

Theorists often view language as a communication system. According to Palmer, language relates something to be communicated with something that communicates, a message on the one hand with 
a set of signs or symbols on the other [11]. Palmer maintains a reservation in classifying language as a communication system. Language is not composed of a message in the form of information, because of the fact that its function's framework is contingent on social relationships. Furthermore, the signified and signifiers' [12] relations in human context is complex, in contrast to the communication system of a traffic light. In these types of communication systems, message can be defined without a language constraint. For example, "RED" means stop.

\subsection{Language as a complex adaptive system}

In the human context, language manifests its characteristic as a social function. This manifestation has two manifolds; cognitive and physical functions which comprise a complex adaptive system (CAS). The system constituents are interacting agents. These agents render the system adaptive because of the fact that agents' behavior is based on past interactions, and current and past interactions together form future possible behavior [13]. The CAS framework renders language a dynamic, generative system rather than a static system of grammatical principles. According to Elis, this system has the following characteristics:

1. The system consists of multiple agents.

2. The system is adaptive; past and present behavior involves forming the future actions.

3. Competing factors affect the agents' behavior.

4. The structure of a language merges from interrelated patterns of experience.

Communication or language in human context can emerge at different points of social interaction. For example, joint actions and cooperative activities all require sharing human beings' intention, culture, with one another [14-16]. Therefore, the CAS approach permits studying language in human context in a way that the aggregated effect of many interacting constraints, including the structure of thought process, perceptual motor biases, cognitive limitations, and social-pragmatic factors [17], are taken into consideration.

\subsection{Pragmatics in communication}

Agents in communication aim to convey the information in a most efficient way. Besides, recognizing the tacit agreement, these agents utilize a variety of operative assumptions in the presupposition framework of the context of communication operation. The significance of these presuppositions is that they do not emerge from the context of the message, rather from contextually formed presuppositions, which is in the agents responsibility [2].

Extracting information is the essential operation in communication. This essentiality ensues from the fact that once the message is imparted, and the extraction (decoding) starts, the sender cannot intervene in this process.

\subsubsection{Context in communication}

The recipient controls the extraction of the information from the imparted message. The sender must in turn be careful in compiling the message to prevent the possibility of misunderstanding. The process of distinguishing, reducing, or even eliminating the pluralism in the constructed meaning of a verbally compiled message is called interpretation [2]. The process of interpretation utilizes context as a reference point. As Rescher says,

in the process of interpretation context is not just important, it is everything. 
Another salient characterization that is attained by context is about assumption and presumptions where both of them are contextual matters. The role of context is a principle of efficiency in communication.

\subsubsection{Communication in communal setting}

A viable and satisfactory communal existence requires that the people understand each other. From individual's point of view, understanding is an endeavor to render the preceding and the proceedings of a rational person, efficiently intelligible to the others in the communal existence. The communality of this rational procedure requires mechanisms such that cognitively confined knowledge can be efficiently probed via communication.

The probing phenomenology is the key element in progression of material science. In fact, human sciences utilize similar notion, however named differently. In human interaction framework, presumptions emerge in a context where humans have questions and seek answers. Yet, presumption is a thought instrument that is employed in circumstances where practical considerations are parameterizing and constraining human cognitive and communicative practices. For example, by changing presumptions, one can overcome a circumstance which lacking of evidence for a conclusive claim that precludes reaching an answer. Rescher's arguments on presumptions are as follows:

Presumption affords useful cognitive and communicative resource. The obvious and evident advantage of presumption as an epistemic device is that it enables us vastly to extend the range of questions we are able to answer. It affords an instrument that enables us to extract a maximum of information from the communication situations. Presumption, in sum, is an ultimate pragmatic resource.

Presumptions are transient. They become substantial in the framework of verbal communication or written communication. Presumptions are contextual and issue driven; a putative fact that is widely employed in communication [2]. Thus, the notion of presumption gains interest in logics, semantics, and epistemology.

The problem stems from the fact that communication limits individual understanding, which is a contingency in making decision in complex environments. Individual limits can be recognized via awareness: a condition of having or showing realization, perception, or knowledge [18].

\section{SITUATIONS THEORY PERSPECTIVE ON UNDERSTANDING}

Situations theory is a meta-theoretical construct that constitutes the cogitation of the circumvent condition of a problem. Therefore, rather developing higher understanding of specific phenomena from well-formulated perspectives, it focuses at understanding how such perspectives contribute to dealing with problems encountered in complex situation [1]. Consequently, here in this section we articulate understanding with in the scope of awareness, which is a situation theory perspective.

\subsection{Situations theory and awareness}

Situations theory facilitates the sustainment of natural ties to reality for any developed construct to understand dynamic, transient, and context-specific situations. Furthermore, situations theory enables the individual involvement of a participant construct rather than a mere observer [1]. This evolution emerged from:

- The incorporation of the participant and maintenance of the observer in the problems [19].

- The practical nature of the problem [1]. 
- The paradigm captured in Pragmatic Idealism that leads to the present state of understanding of situations theory [20].

The salient evolution for situations theory towards awareness entailed from the seminal work Pragmatic Idealism [20]. As an ontological construct, the formation of awareness is articulated in the Representation of Reality - RDP model.

\subsubsection{Reality, domain, and perspective - RDP model}

In defining a perspective $\mathrm{P}$, a state of awareness is required. Establishment of a state of awareness is an extremely complex problem. The first issue is whether for awareness to exist we first need awareness of self, or whether we need awareness of other than self. To circumvent any debate of primacy, we view this as a mutually generative process (GP). The self defines that which we perceive and that which is perceived ultimately defines the self. Due to the imposition of the self on awareness, it must be recognized that every domain of awareness will in essence be unique, its formation defined by a person's dispositions, past experiences, and even future expectations. For instance, in an inquiry about another domain of awareness, the presuppositions ensued from these formations. The imposition of other than self on awareness will, however, also means that the perspectives, irrespective of individual interpretation, may share commonalities.

To be able to share perspectives in their totality; that is to say there is a sharing of meaning not only of information, two agents must share awareness. This produces an interesting dilemma. The assertoric nature of the formation process of each state of awareness would mean that sharing should not be possible. We, however, know this not to be true. To overcome this dilemma, the nature of awareness, and particularly the manner in which the domain of awareness is established must be studied further.

\subsubsection{Awareness and the domain}

The formation of a domain takes place through the GP. The GP inculcates the mutual generation of self-awareness and awareness of other than self (Fig. 1). The formalization of all that is other than self will at that point be captured as the domain of awareness. It is the basis of the situational construct; observer, entity, and solution form. Typically, complexity is a feature that results from the misalignment or tensions that exist between these three elements.

The domain of awareness plays a critical role in our ability to understand a problem. It captures the degree to which a problem may be comprehended. The degree to which abstractions in the form of simplifications and assumptions must be made will affect comprehension. The effectiveness of the domain of awareness can be represented by this abstraction in a nominal construct called the abstraction distance.

3.1.3 Comprehensibility, understanding, and complexity

For conditions where the domain is a crude approximation of the problem, as represented by the conceptual approximation distance $\mathrm{A}^{\prime}(\mathrm{d})$, the possible comprehension will be low. Conversely high comprehension requires a low approximation distance, where $\mathrm{A}^{\prime}(\mathrm{d}) \rightarrow 0$. A perspective is formed within a domain of awareness. The perspective represents that which is understood of the problem. The perspective is itself an abstraction of the domain of awareness. Therefore, an additional abstraction distance $\left(\mathrm{A}^{\prime \prime}(\mathrm{d})\right)$ can be postulated that references the degree to which that which may be comprehended is understood. In this model, the complexity is a function of the effectiveness of what is understood. What is understood is the basis on which action is taken. Action is broadly defined to encompass any resultant activity. This may include a decision, a design, an explanation, 


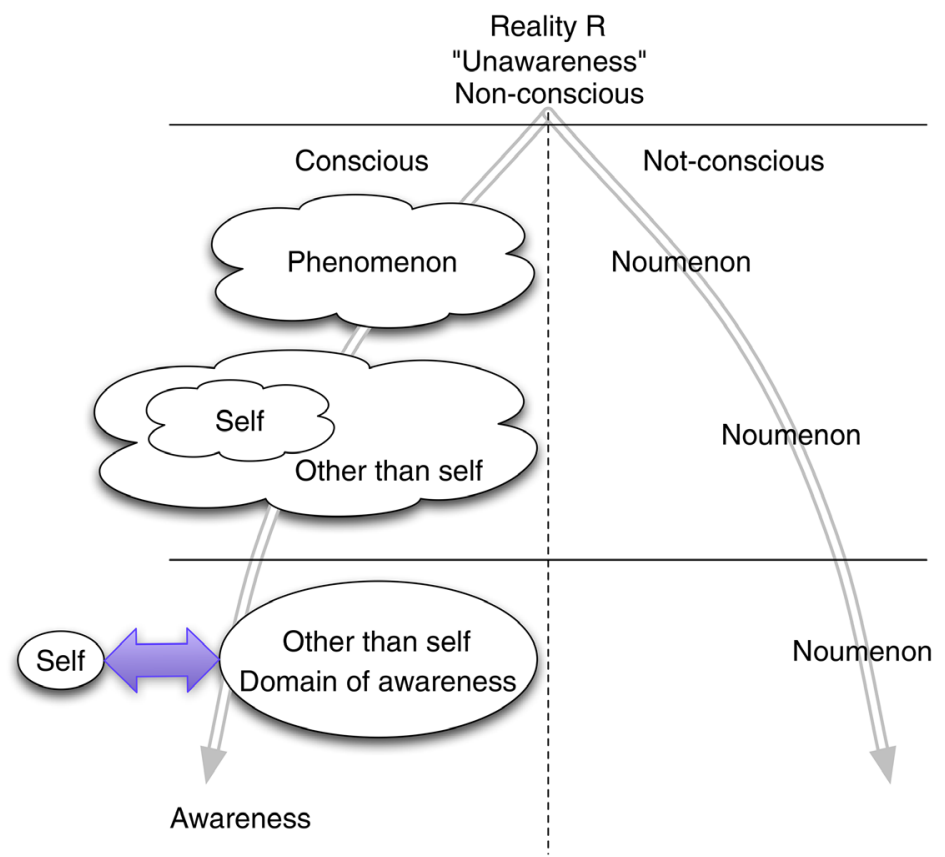

Figure 1: Construction of self and other than self. Noumenon represents unbounded participation of human agent, and phenomenon represents cognitive and bounded observation of life.

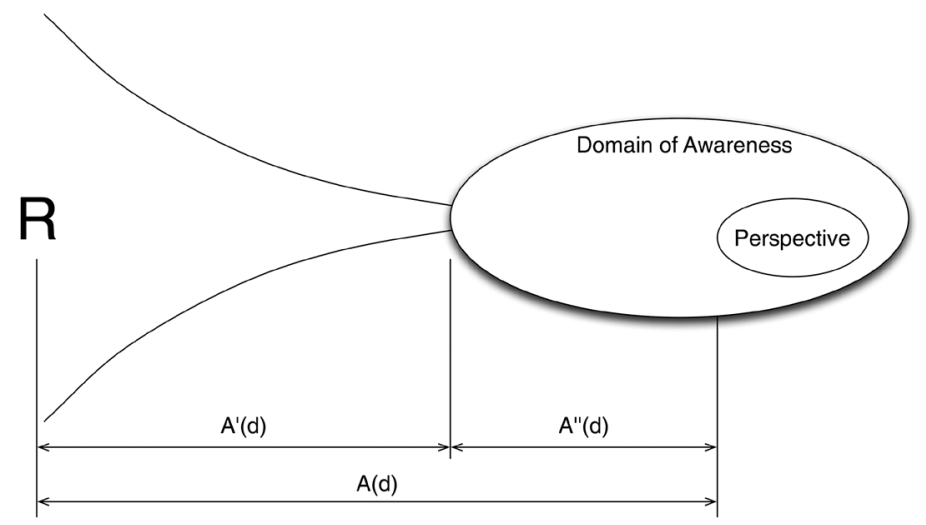

Figure 2: Domain of awareness and perspective with respect to realty.

or purposefully doing nothing. Notionally, action is derived from what are broadly referred to as propositional statements, or claims. Determinations of whether that which is understood is 'right' or 'wrong' can in a very rudimentary manner be defined by whether a claim is ultimately reflective of what transpires. The overall complexity of a situation that can be symbolized by $\mathrm{A}(\mathrm{d})$ is a combination of $\mathrm{A}^{\prime}(\mathrm{d})$, and $\mathrm{A}^{\prime \prime}(\mathrm{d})$ (Fig. 2).

In the complex situation, $A(d)>>0$. The effect of this is that the perspective, which will be consistent with the domain of awareness, will only generate understanding of the problem that is 
extremely limited. This does not mean that a claim that is made will be wrong. The probability that the claim will be erroneous $(\mathrm{CL}(\varepsilon))$ will [20], however, increase as A(d) increases. This does introduce a complication on the notions of right and wrong. Since in a complex situation, we should expect A(d) to always be high; the probability of making erroneous claims will always be high. In other words, the chances of being wrong are extremely high. A distinction in probability of making erroneous knowledge claims can be made based on whether the source is due to $A^{\prime}(d)$ or $A^{\prime \prime}(d)$.

\subsubsection{Generative process}

The dynamics of GP that are incorporated into the situations theory renders the construction of self and other-than-self distinguishable from other domains and perspectives. The GP is how we come about forming a domain of awareness. At the highest level of resolution, every perspective will be completely unique based on the uniqueness of the components that form the GP (Figs 1-3).

The differences between two domains may lie not in the comprehension that they provide, but how that comprehension is generated. The generation process includes assertoric claims. These claims made by two domains may be the same, but not acceptable to each other based on how the claims were generated and how they are justified. The GP might be likened to a 'black box process' in which we know we are moving from a state of unbounded, non-conscious, complete connectedness with Reality to a state in which we have a bounded, cognitive, conscious perspective of Reality. The components in Fig. 3 can be summarized as; past experiences, coping strategies developed, and cognitive dispositions and cognitive predispositions.

\subsubsection{Situational awareness}

The SA definition provided by Endsley [21] renders situational awareness and integration of both process and product where the distinction between these two is that; (i) state of awareness representing the information and knowledge is product; (ii) perceptual and cognitive activities involved in constructing, updating, revising the state of awareness [22].

In this regard, the situational awareness is a construct of participant-observer duality that incorporates the integrated meaning stemming from spatial-temporal constraints, goals/purpose,

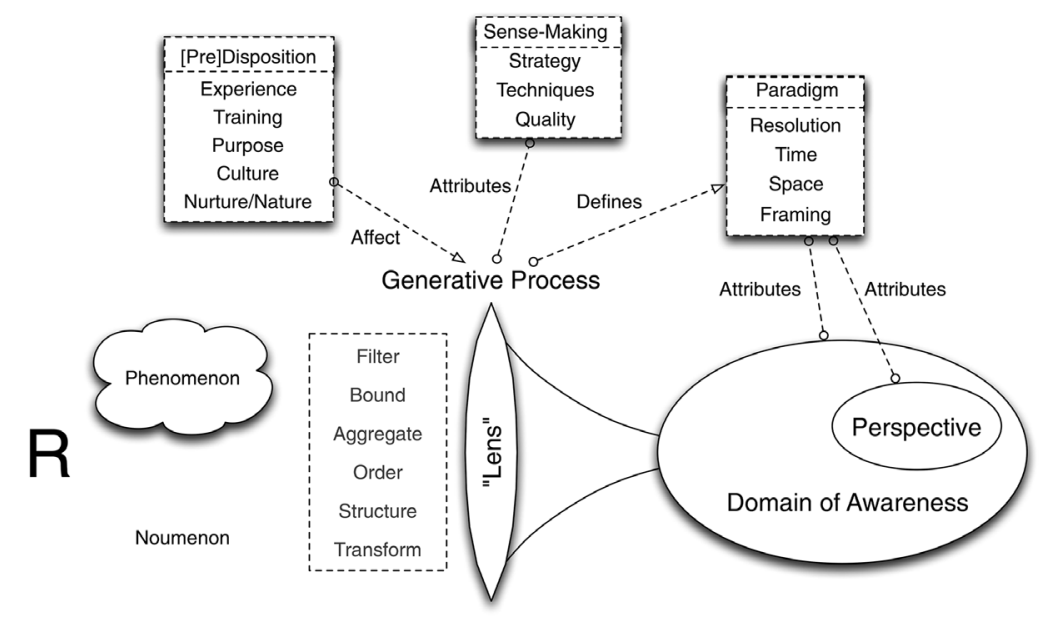

Figure 3: Components of generative process in the construction of a domain of awareness (is that which is comprehended) and perspective (is what is understood). 
understanding of the situation, perceived mental models, and classification of the situation. These are manifested in the interaction between the situation and datum in the construct of situational awareness. Since situation awareness, as a state of knowledge, is distinguishable from the GP [23], utilizing different GPs does not preclude maintaining common situational awareness. Furthermore, this GP constitutes a cogent model that allows anticipation and interpretation of the available information within the domain of awareness [24].

3.1.6 Shared situational awareness

The Situations Theory framework invokes the cognitive representation of reality (CRR) [25] for the representation of the cogent aspect of situational awareness. The significant contribution of the CRR concept is the shift from mere cognitive awareness to domain of awareness by utilizing the GP. Along with the RDP approximation, CRR (principle of awareness as the disassociation) approach mitigates constraints that emerge from awareness and knowledge relation. Therefore, any knowledge statement will have a degree of fallibility $[18,20,25]$ that is inferential in a context that constitutes a new paradigmatic new worldview, generalizations of concepts, and placing the impact of participation into the situation. In this context, interaction of particulate domain of awareness becomes the medium for shared awareness [18] to emerge by utilizing purposeful information exchange.

\subsubsection{Required condition for sharing}

The a priori parameters required for the construction of congruent domains of awareness supporting conditional sharing include:

1. Link

2. Intentionality/imperative

3. Orthodoxy

4. Factors that affect the conveyance of meaning, or the establishment of congruent meaning a. Disposition

5. Transfer of factors influenced by disposition

b. Signifiers and context

c. Elements of the GP; how the context was formed, factors in the framing that will influence the manner in which interpretation took place

6. Metadata
d. Time
e. Place
f. Nature of situation

In the simplest case where there is a link, intent, and no orthodoxy, this problem is reduced to a study of dispositions. How the claim was established by agent 1 , and whether the claim is comprehensible, and can be understood by agent 2 .

\subsubsection{Type of shared awareness}

Based on the General Theory of Shared Awareness [18], four general strategies for sharing are proposed depending on whether the shared space that is to be established will be derived from, or require conditional sharing, contextual sharing, synthetic sharing, or synoptic sharing. In this article, communication is studied only in the conditional sharing framework. 


\subsection{Shift in semantic to pragmatic meaning}

Meaning is an emergent attribute of a message. Meaning is generated from the interaction of components of the message. The components will be tacit and explicit. The message will include content elements in the form of signifiers. Meaning is derived from the manner that the signifiers are structured together and interact within a context. The context will include a variety of components including: The manner in which the message is conveyed, the manner in which the sender formulated the perspective from which the message is being generated, and even the manner that the sender expects the receiver to interpret or decode the message.

\subsubsection{Truth meaning vs. used meaning}

The meaning analysis of a statement in the context of linguistic is confined within the appropriateness and correctness of usage. Thus, the relation with real meaning is not commercial to articulate in communication models [26].

Communication generates integrated meaning that is contingent on context, time, predisposition, orthodoxy, etc. Since each of these contingencies renders the meaning idiosyncratic, RDP model can enhance communication model with distinguishing the truth meaning and used meaning where "truth" and "used" meaning defined as [26]:

$\mathrm{u}_{\mathrm{nm}}\left(\mathrm{SA}_{\mathrm{i}}, \mathrm{P}_{\mathrm{nm}}\right)=$ the information shared situation SA satisfies the use condition for the claim $\mathrm{P}$,

$t_{n m}\left(S_{i}, P_{n m}\right)=$ the informative situation $S$ satisfies the truth condition for the claim $P$.

Learning the meaning of a word (attaining an understanding/awareness of it) is a regimen of learning how it is used. Therefore, a shift in the meaning is possible that manifests itself in communication in the domain of awareness. This will take the form of discrepancies in the shared understanding that is established and will ultimately qualitatively influence shared/common decisions.

\subsubsection{Communicating in conditional shared situational awareness}

From a natural decision-making point of view, "choosing among alternatives" is elaborated as attaining a goal through reaching a common perspective. Shared awareness is a state of comprehension for a population that is functionally dependent on the establishment of shared awareness between agents. This form of sharing articulate one manner in which perspectives built on heterogeneous paradigms can form a conditional shared construct. In conditional sharing, one agent moves towards another agent's domain of awareness. Extrapolating the communication framework, the sender is viewed to be dominant. The sender is notionally viewed to have the desire, and predisposition to move the domain of awareness.

In Fig. 4, this is depicted in the RDP model. There are two domains of awareness, $D_{1}\left(t_{n}\right)$ and $\mathrm{D}_{2}\left(\mathrm{t}_{\mathrm{n}}\right)$, multiple perspectives in these two domains of awareness $\mathrm{P}_{\mathrm{Dn}_{n}}$. The approximation distance to reality (R) for perspective is $A_{D}(d)$, for domain of awareness is $A_{D}^{\prime}(d)$, and perspective with respect to domain of awareness is $A^{\prime \prime}{ }_{D}(d)$. The separation between two domains of awareness is $A_{s}\left(d, t_{n}\right)$. There is a signifier domain for verbal communication where the constructed presumptions (= perspective) represent a meaning. This is indeed where the RDP model becomes important. According to the earlier definition of complex situation in [20], complexity is defined as "making an erroneous knowledge claim CL(E)". The truth meaning $-\mathrm{t}_{\mathrm{nm}}\left(\mathrm{S}_{\mathrm{i}}, \mathrm{P}_{\mathrm{nm}}\right)-$ is in the reality. When an agent initiates an approximation by making a claim of

$$
\mathrm{CL}_{\mathrm{i}}(\varepsilon) \cdot \mathrm{t}_{\mathrm{nm}}\left(\mathrm{S}_{\mathrm{i}}, \mathrm{P}_{\mathrm{nm}}\right) \equiv \mathrm{u}_{\mathrm{nm}}\left(\mathrm{SA}_{\mathrm{i}}, \mathrm{P}_{\mathrm{nm}}\right) .
$$




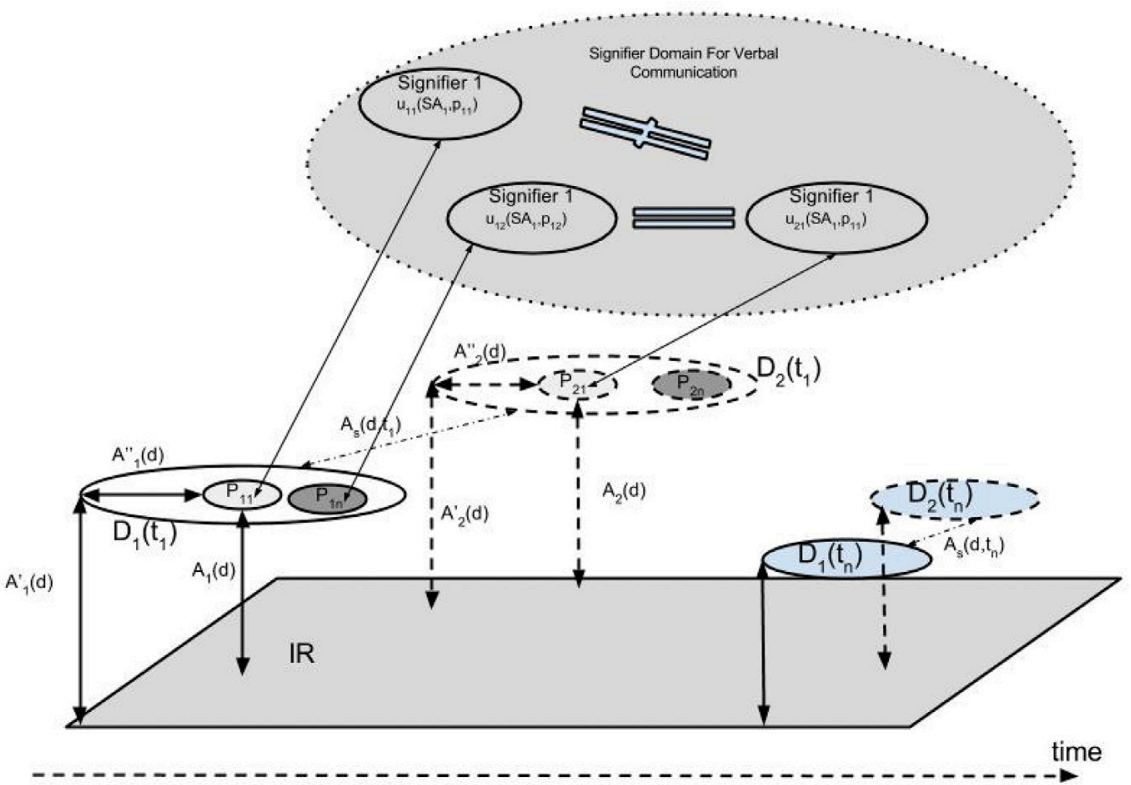

Figure 4: RDP representation of communication in shared awareness and communication.

Based on this construct, the integrated meaning is constrained by the intrinsic process. Explicitly, however, substantiation of the words is the same, which precludes attaining a shared decision.

The desire for sharing for the sender's domain, $\mathrm{D}_{1}$, entails further $\mathrm{CL}_{\mathrm{i}}(\varepsilon)$ so that the same used meaning attained with move towards perspective in $\mathrm{D}_{2}$

$$
\mathrm{CL}_{\mathrm{i}}(\varepsilon) \cdot \mathrm{u}_{\mathrm{nm}}\left(\mathrm{SA}_{\mathrm{i}}, \mathrm{P}_{\mathrm{nm}}\right) \equiv \mathrm{u}_{\mathrm{nm}}\left(\mathrm{ShSA}_{\mathrm{i}}, \mathrm{P}_{\mathrm{nm}}\right) .
$$

This generates a shift in meaning from semantic to pragmatic to attain a decision. This algedonic loop that constitutes $\mathrm{CL}_{\mathrm{i}}(\varepsilon)$ iterations to the used meaning of the sender occurs so that the separation between the two domains of awareness $\mathrm{A}_{\mathrm{s}}\left(\mathrm{d}, \mathrm{t}_{\mathrm{n}}\right)$ becomes $\mathrm{A}_{\mathrm{s}}\left(\mathrm{d}, \mathrm{t}_{\mathrm{n}}\right) \rightarrow 0$.

\section{CONCLUSION}

The meaning of a word in linguistic framework is rooted in semantics, hence dominated over used meaning. However, the real meaning of a word can be understood possibly better with what come after it. Studying communication in situations theory perspective allows recognizing the proper causes of discrepancies in communal understanding. The pragmatic meaning, manageable, is tenable to overcome knowledge discrepancies that might causal emerge from different sources while constructing understanding. General Theory of Shared Awareness permits studying the communication in this framework so that predispositions and cultures involved can be preserved and adaptation required environments are sustained. 


\section{REFERENCES}

[1] Sousa-Poza, A., A narrative of [complex] situations and situations theory. Managing and Engineering in Complex Situations, Vol. 21, eds. S.F. Kovacic \& A. Sousa-Poza, Springer: The Netherlands, pp. 13-44, 2013.

[2] Rescher, N., Communicative Pragmatism, Rowman \& Littlefield Publishers, Inc.: Lanham, 1998.

[3] Huseman, R.C., Lahiff, J.M. \& Hatfield, J.D., Interpersonal Communication in Organizations, Holbrook Press: Boston, MA, 1976.

[4] Chapanis, A., Men, machines and models, American Psychologist, 16, p. 115, 1971.

[5] Lasswell, H.D., The structure and function of communication in society. The Communication Ideas, ed. L. Bryson, Institute for Religious Studies and Socia Studies: New York, 1948.

[6] Shannon, C.E. \& Weaver, W., The Mathematical Theory of Communication. University of Illinois Press: Champaign, IL, 1949.

[7] Berlo, D.K., The Process of Communication, Holt, Rinehart \& Winston: New York, 1960.

[8] Barnlund, D.C., A transactional model of communication, Language Behavior: A Book of Readings, eds. A.G. Johnnye Akin, G. Myers, J. Stewart, Mouton \& Co. N. V.: Hague, 1970.

[9] Hall, A.D. \& Fagan R.E., Definition of system. General System 1, pp. 18-28, 1956.

[10] Paul Watzlawick, J.B.B. \& Jackson, D.D., Pragmatics of Human Communication: A Study of Interactional Patterns, Pathologies and Paradoxes, Norton: New York, 2011.

[11] Palmer, F.R., Semantic: A New Outline, Cambridge University Press: London, 1976.

[12] De Saussere, F., Course in General Linguistic, McGraw-Hill: New York, 1959.

[13] Elis, N.C., Language is a complex adaptive system. Language As a Complex Adaptive System, Santa Fe Institute, 2007.

[14] Bratman, M., Shared cooperative activity. The Philosophical Review, 101, pp. 327-341, 1992. doi: http://dx.doi.org/10.2307/2185537

[15] Bratman, M., Shared intention. Ethics, 104, pp. 97-113, 1993. doi: http://dx.doi. org/10.1086/293577

[16] Clark, H.H., Using Language, Cambridge University Press: Cambridge, 1996. doi: http://dx.doi. org/10.1017/CBO9780511620539

[17] Christiansen, M.H. \& Chater, N., Language as shaped by the brain. Behavioral \& Brain Sciences, 31, pp. 489-558, 2008. doi: http://dx.doi.org/10.1017/S0140525X08004998

[18] Kovacic, S., Micro to macrodynamics of shared awareness emergence in situations theory: towards a general theory of shared awareness. Engineering Management and System Engineering, Old Dominion University, 2013.

[19] Sousa-Poza, A., Kovacic, S, \& Keating, C., System of systems engineering: an emerging multidiscipline. International Journal of System of Systems Engineering, 1, pp. 171-188, 2008.

[20] Sousa-Poza, A. \& Correa, Y., Pragmatic idealism as the basis for understanding complex domains: the trinity and SOSE, IEEE SMC, Hawaii, 2005.

[21] Endsley, M.R., Design and evaluation for situation awareness enhancement. Human Factors Society 32nd Annual Meeting, Santa Monica, CA, 1988.

[22] Adams, M.J., Tenney, Y.J., \& Pew, R.W., Situation awareness and the cognitive management of complex systems. Human Factor, 37(1), pp. 85-104, 1995. doi: http://dx.doi. org/10.1518/001872095779049462 
[23] Endsley, M.R., Toward a theory of situation awareness in dynamic system. Human Factor, 37(1), pp. 32-64, 1995. doi: http://dx.doi.org/10.1518/001872095779049543

[24] Neissser, U., Cognition and Reality: Principles and Implications of Cognitive Psychology, W.H. Freeman and Company: San Francisco, CA, 1976.

[25] Brewer, V.E., A decision making construction for complex situations.. Engineering Management and System Engineering, Old Dominion University, 2010.

[26] Rescher, N., Meaning and assertability. Empirical Inquiry. Rowman \& Littlefield: Totowa, NJ, 1982.

[27] Honderich, T., The Oxford Companion to Philosophy, Oxford University Press: New York, 1995. 